\title{
Application of Models from Epidemiology to Metrics for Computer Virus Risk
}

\author{
J. L. Aron
}

Science Communication Studies

5457 Marsh Hawk Way, Columbia, Maryland 21045, USA

Tel: 410-740-0849, Fax: 410-964-3598, Email: jaron@mstec.org

\section{R. A. Gove}

Science Applications International Corporation

8301 Greensboro Drive MS E-12-6, McLean, Virginia 22102, USA

Tel: 703-748-4208, Fax: 703-356-6961,

Email: Ronald.Gove@cpmx.saic.com

\begin{abstract}
One aspect of maintaining integrity in information systems is establishing an organizational environment that will prevent the damage caused by external agents. One particularly insidious such agent is the computer virus. It can alter data often without the owner or user of that data being aware. Establishing such an environment can often rely on the availability of metrics for organizational characteristics associated with harm to data integrity. This paper will focus on the development of organizational metrics for the threat of computer viruses. It is expected that many of these metrics will apply to other threats to data integrity although we have not pursued that line of research.

In the case of computer viruses and some other types of malicious code, the formal analogies to the infection dynamics of biological viruses permit the utilization of epidemiological concepts in the development of metrics. This paper demonstrates how a simple epidemiological model of computer viruses provides insights about the importance of several metrics:
\end{abstract}

- frequency of contact;

- utilization of antiviral software; 
- effectiveness of antiviral software;

- likelihood of notifying other people about computer viruses detected;

- frequency of updating antiviral software.

The dynamics of computer virus transmission will be combined with broader environmental factors of an organization in a computer simulation model that elaborates on the simple one shown here. In addition, epidemiological concepts from environmental health permit the integration of environmental perspectives and disease control. This paper shows how these concepts are guiding the development of survey methods to obtain information for the construction and validation of the computer simulation model. The benefits of a broader analysis of an organization's environment are an understanding of how organizational complexities influence the risk associated with computer viruses, a representation of organizational factors in terms familiar to those making decisions and integration of assessment of risk from multiple threats.

Strengths and weaknesses of various methodologies are discussed. Looking beyond the current project, future research is suggested in the areas of integrated assessment modeling, data collection strategies, and the threats presented by new kinds of malicious code.

\section{Keywords}

Mathematical model, risk assessment, computer virus, malicious code, computer simulation, information security metric

\section{INTRODUCTION}

One aspect of maintaining integrity in information systems is establishing an organizational environment that will prevent the damage caused by external agents. One particularly insidious such agent is the computer virus. It can alter data often without the owner or user of that data being aware. Establishing such an environment can often rely on the availability of metrics for organizational characteristics associated with harm to data integrity. This paper will focus on the development of organizational metrics for the threat of computer viruses. It is expected that many of these metrics will apply to other threats to data integrity although we have not pursued that line of research.

Paradoxically, computer viruses may be scarcely mentioned in an overview of issues in information security and integrity management (GAO, 1998), while a large percentage of organizations report computer virus incidents despite widespread use of antiviral software (Power, 1998). The proper assessment of computer viruses in information security and integrity management depends on estimates of the risk and impact of computer virus incidents and an analysis of how they are influenced by various factors in the computing environment. Since 
relatively little good data exist, the methods for risk assessment include mathematical or computer simulation models to synthesize available information and provide a theoretical basis for the development of metrics. Development of metrics in turn can guide additional data collection efforts.

The first step is to use formal analogies with the transmission of biological viruses. The use of these analogies is justified as mathematical formulations of propagation processes that are not restricted to biological agents and indeed can be applied to various physical phenomena. In the early 1990s, there was a flurry of interest in models of the dynamics of the transmission and control of computer viruses (NCC, 1991). The best example of this work is from the IBM research labs (Kephart and White, 1991; Kephart and White, 1993). This work offers a compelling explanation of the observation that computer viruses never became a problem for all (or nearly all) computers in all organizations. Much of the subsequent effort in this area has been directed at the development of better software tools to control viruses (Kephart et al, 1997). However, in the midst of a growing recognition of the importance of databases for information security and integrity management (GAO, 1998), there is a need to embed the concepts from computer viruses in a broader framework of organizational metrics.

Section 2 demonstrates how a simple epidemiological model of computer viruses provides insights about the importance of measures of (i.e., 'metrics') for frequency of contact, utilization of antiviral software, effectiveness of antiviral software, likelihood of notifying other people about computer viruses detected, and frequency of updating antiviral software. The dynamics of computer virus transmission will be combined with broader environmental factors of an organization in a computer simulation model that elaborates on the simple one shown here. Epidemiological concepts from environmental health permit the integration of environmental perspectives and disease control. Section 3 describes the organizational factors considered in the development of survey methods to obtain information for the construction and validation of the computer simulation model. Finally, Section 4 discusses the strengths and weaknesses of the modeling approach and the survey design. Topics for future research are suggested in the areas of integrated assessment modeling, data collection strategies, and the threats presented by new kinds of malicious code.

\section{SIMPLE EPIDEMIOLOGICAL MODEL OF COMPUTER VIRUSES}

The aim of the research discussed in this paper is to develop a mathematical model that will characterize individual outbreaks of computer viruses in an organization of computer users whose computers define a population of interest. The simple model presented here illustrates basic principles for the control of computer viruses and forms the basis for a more detailed computer simulation model that will be used to construct organizational metrics. 
An individual outbreak is caused by the replication of a single computer virus. A computer is infected and infective when the single computer virus that could be transmitted to another computer is in the computer's memory or an application program on its hard drive. Immunity in this context means that antiviral software installed on a computer can be used effectively for detection and prevention of infection. Immunity has to be considered separately for specific viruses because antiviral software may be effective against some viruses and not others. The term partial immunity is used because computer viruses that can be detected by installed antiviral software are sometimes missed because the software is not always used effectively. If the antiviral software cannot detect the computer virus at all, then the virus is considered to be undetectable.

The basic reproduction ratio is a useful metric from epidemiology that represents the potential spread of infection from a single infected individual. The basic reproduction ratio characterizes threshold behavior of the dynamics of transmission. If the ratio is less than unity, then the infection does not spread. If the ratio is greater than unity, the infection does spread. Larger values of the ratio indicate a greater amount of spread. The basic reproduction ratio is the product of contacts per day, the duration of infection, and the fraction of contacts that are susceptible. The structure of the model and the results are described in more detail in the Appendix.

The parameter values are intended to be illustrative. That the results are sensitive to these values demonstrates that these parameters are good candidates for organizational metrics. The analysis of the basic reproduction ratio in Table 4 makes clear that transmission will be increased not only in organizations with higher rates of contacts that permit transmission of computer viruses, but also with organizations in which infections remain undetected for longer periods. The beneficial effect of the notification policy in Table 4 is a result of people acting promptly to notify system administrators of computer virus problems and reduce the period in which infections remain undetected. The basic reproduction ratio can be readily calculated for any choice of parameters.

However, the calculation of outbreak sizes from the basic reproduction ratios is not so simple. Although the general relationships among the outbreak sizes in Table 2 are explained by the basic reproduction ratios in Table 4 , it should also be noted that the outbreak sizes are somewhat smaller overall than might be expected from the corresponding basic reproduction ratios. The reason for the difference is the assumption that the first detection of an infected computer stops the outbreak. As more computers are infected in the outbreak, there is a greater chance that at least one of the infected computers will be detected. Consequently, the outbreak is usually terminated before the initial infection completes the duration of infection expected if it were the only infection. In general, computer simulations are needed to characterize the effects of complex organizational behavior like patterns of reporting.

Another issue is the effect of updating software. A major concern is the generation of new computer viruses. The frequency of updating antiviral software is another 
important organizational metric. Clearly, large numbers of computers can be infected if antiviral software cannot detect a computer virus (see the undetectable scenario in Table 2). In this scenario, all of the computers are considered to be susceptible with no immunity at all. Fortunately, a large majority of incidents are caused by a small number of computer viruses (NCSA, 1996). Although new computer viruses are generated all the time, very few computer viruses reach large numbers of computers. Therefore, the percentage of exposures to computer viruses that result in the undetectable scenario is expected to be small. The percentage of such exposures can and should be reduced by regular updates of antiviral software, but a focus on increasing the frequency of updating to higher and higher levels will have a progressively smaller effect on the number of computer virus incidents. The problem of undetectable computer viruses is that of relatively rare but highly damaging events.

\section{RISK FACTORS IN ORGANIZATIONAL ENVIRONMENT}

The dynamics of computer virus transmission will be combined with broader environmental factors of an organization in a computer simulation model that elaborates on the simple one shown above. Epidemiological concepts from environmental health permit the integration of environmental perspectives and disease control (Beaglehole et al, 1993). These concepts are guiding the development of survey methods to obtain information for the construction and validation of the computer simulation model. Computer security metrics are analogous to environmental health indicators, which are defined as an expression of the link between environment and health, targeted at an issue of specific policy or managenent concern and presented in a form which facilitates interpretation for effective decision-making (Briggs et al, 1996). An event, condition, characteristic or combination of such may be associated with a disease in that the presence of a factor indicates an increased risk of occurrence of a disease. Establishing associations is an important part of linking the environment with disease. In applying these concepts to computer security, adverse security outcomes replace adverse health outcomes. The benefits of a broader analysis of an organization's environment are an understanding of how organizational complexities influence the risk associated with computer viruses, a representation of organizational factors in terms familiar to those making decisions and integration of assessment of risk from multiple threats. Therefore, the pertinent information about an organizational environment includes parameters specific to computer viruses and characteristics of the security environment in general.

We are fielding a survey in 1998 via the World Wide Web with the focus at the level of a workgroup because the security practices of workgroups may be quite heterogeneous in a large organization. The survey has several sections, including: 
- basic demographic information about the organization and the workgroup of the respondent;

- views of the respondent about the threat of computer viruses and general aspects of protection;

- system environment including groupware, network management and organizational turnover;

- practices for sharing files, ranging from physical media to shared network volumes and internet services

- practices for system protection, ranging from URL filtering, usage of antiviral software, reporting of computer viruses to system administrators to user training about information security.

The environmental variables are being analyzed in relation to variables about computer virus experience over the past twelve months in the respondent's workgroup. Experience takes into account not only the frequency of occurrence of a computer virus incidents, but also the impact of such incidents on the organization. In addition to questions about recent experience, questions are asked about serious computer virus incidents at any time, where the respondent is told that an incident could be serious because of size, duration, information lost or activities disrupted. It is desirable to ask about serious computer virus incidents because many computer virus incidents have limited impact and therefore low priority as a security issue. However, it is necessary to ask about serious computer virus incidents with a longer time frame because such incidents are relatively rare. Although respondents are asked about factors that were responsible for the most recent serious computer virus incident and its impact, it is not possible to analyze these incidents in relation to all of the other environmental variables because of the longer time frame and possibly different location.

\section{DISCUSSION}

This paper builds on models of the epidemiology of computer viruses, especially the work from the IBM research lab from the early 1990s (Kephart and White, 1991; Kephart and White, 1993). Like the earlier models, there is a basic threshold of contact required for spread of infection that depends on how long infections remain undetected. Notification policies can be very effective at reducing transmission even though the policies in different models are implemented quite differently.

The most important difference is the assumption about the pattern of contact. The IBM work assumes that transmission patterns are tightly clustered whereas the model in Section 2 and the Appendix assumes a simple pattern of random contact. The difference arises in part because of the different aims of the models. The aim of the IBM study was to make general statements about the phenomenon of clustering of contacts in small groups. The assumption of tight clustering leads to the 
conclusion that the prevalence of computer viruses will rise slowly even in the absence of antiviral protection, although antiviral protection does help reduce the prevalence. Occasional large outbreaks are not a contradiction; the aim is an examination of all susceptible computers in the population. However, there was no attempt to collect detailed data on the patterns of contact that would be required to construct security metrics for particular workgroups. For this purpose, it is desirable to keep the model structure and associated data requirements as simple as possible.

Another reason for the differences in the assumptions about contact patterns is the different time periods involved. Highly clustered contact patterns are probably less important in limiting transmission of computer viruses in the computing environment of the late 1990s because several factors in recent years have increased opportunities for transmission. There is more electronic mail allowing attachments, intranet servers allowing ready access to documents across an organization, as well as the explosive growth of the internet. Associated organizational changes foster more contact across and within organizations. Workers are shuffled in and out of flexible teams while more electronic communication is shared between clients and vendors. It remains to be seen if a model structure based on random contacts produces a reasonable estimate of computer virus risk. The answer will probably depend on the size of the workgroup, with reasonable estimates for smaller groups and gross overestimates for large organizations. It is also difficult to treat large organizations as a unit because of heterogeneity in security practices within organizations. The appropriate linkage of security issues at different levels of the organization requires more attention.

The development of models for integrated assessment also demands a greater connection between the studies of the spread of computer viruses and the organizational environment. In order to achieve this integration, it is necessary to collect relevant data from organizations. Little or no useful data exist that correlate specific losses to the integrity or confidentiality of computer data to specific incidents. One of the more detailed computer virus surveys in the U.S. that is publicly available is an annual telephone survey of organizations conducted by the National Computer Security Association (now the International Computer Security Association). This survey, whose script is provided elsewhere (NCSA, 1996), contributes useful information about the types of viruses in circulation, routes of entry into the organization and types of antiviral software. However, the survey being fielded by us has more organizational information such as attitudes towards computer virus security, general information security training and turnover in the organization. To some extent, this reflects the difference between a focus on specific brands of technology products and a focus on the organizational environment; it is probably impossible to do justice to both areas in one survey.

The General Accounting Office has found a growing interest in more precise measurement of costs and benefits of security-related policies (GAO, 1998). Better characterization of the severity of security and integrity incidents is clearly needed. 
A recent study of Internet security incidents reported to the Computer Emergency Response Team at Carnegie Mellon University combined severity measures related to duration, the number of sites involved and the number of messages generated (Howard, 1997). Research on impacts will have to integrate computer virus issues with other security issues because of the crosscutting effects of security policies. For example, investment in better backup services has to be justified on the basis of all threats to data integrity.

In addition to generating databases for internal management, there has to be an effort to overcome barriers to external reporting of computer security information. Examination of security problems across organizations provides important insights about threats and the effective organizational strategies. Tools that allow such information to be gathered while maintaining confidentiality are a challenge.

Finally, any effort to develop metrics for computer virus risk must be aware of the evolving threat. The nature of the computer virus threat has already changed as the predominant operating systems have changed (Kephart et al, 1997). In the future, there may be new hybrids of computer viruses with malicious code that slips in through inadvertent access of URLs because of the trend of becoming unaware of or indifferent to the physical location of accessed files. This might be in the metadata of a Microsoft Word document or the HTML in electronic mail. Computer viruses could attack software agents that are authorized to send electronic mail with attachments, in some ways similar to the Internet worm that rapidly made copies of itself and sought new hosts (Kephart et al, 1997). At another level, evolutionary computational models allow programs themselves to evolve in ways analogous to natural selection, removing the human programmer from the loop (Burke, 1998). Clearly, integrated assessment of computer virus risk has to be embedded in a larger context of malicious code and information security.

\section{ACKNOWLEDGMENTS}

We appreciate the assistance of Cristina Schneider and Jon McKnight in the development of this project as well as the efforts of Myron Cramer, Cedric Armstrong and Jim Frazer in earlier phases. This work was supported by the U.S. government under contract MDA 904-97-C-D618.

\section{REFERENCES}

Beaglehole R., Bonita R. and Kjellstrom T. (1993) Basic Epidemiology. World Health Organization, Geneva.

Briggs D., Corvalan C. and Nurminen M. (1996) Linkage Methods for Environment and Health Analysis. General Guidelines. World Health Organization, Geneva. 
Burke, D.J. (1998) Evolvability of Emerging Viruses, in Pathology of Emerging Infections 2 (eds. A.M. Nelson and C.R. Horsburgh, Jr.), American Society for Microbiology, Washington DC (in press), Chapter 1.

GAO. (1998) Executive Guide. Information Security Management: Learning from Leading Organizations (GAO/AIMD - 98 - 68). General Accounting Office, Washington, DC.

Howard J.D. (1997) An Analysis of Security Incidents on the Internet 1989-1995. Ph.D. Dissertation in Engineering and Public Policy, Carnegie Mellon University, Pittsburgh, Pennsylvania.

Kephart J.O. and White S.R. (1991) Directed-graph epidemiological models of computer viruses, in Proceedings of the IEEE Computer Society Symposium on Research in Security and Privacy, Oakland, CA, May 20-22, 1991, 343-359.

Kephart J.O. and White S.R. (1993) Measuring and modelling computer virus prevalence, in Proceedings of the 1993 IEEE Computer Society Symposium on Research in Security and Privacy, Oakland, CA, May 24-26, 1993, 2-15.

Kephart J.O., Sorkin G.B., Chess D.M. and White S.R. (1997) Fighting computer viruses. Scientific American. 277(5), 88-93.

NCC. (1991) Proceedings of the Fourth Annual Computer Virus \& Security Conference, New York City, March 14-15, 1991. Nationwide Computing Corporation.

NCSA. (1996) NCSA Virus Study. Complete Results \& Analysis, National Computer Security Association (now International Computer Security Association), Carlisle, Pennsylvania.

Power R. (1998) 1998 CSI/FBI Computer Crime and Security Survey, Computer Security Issues \& Trends, Computer Security Institute, San Francisco, California,4(1), 1-12.

\section{BIOGRAPHIES}

Joan L. Aron earned her Ph.D. in mathematical biology at Princeton University and has worked on applications of mathematics in public health at the National Institutes of Health and at the Johns Hopkins School of Public Health. She is now President of Science Communication Studies, a nonprofit organization for crossdisciplinary studies. Its primary focus is mathematical modeling and database design for environment and health issues, especially infectious diseases. Other areas of interest are the application of public health principles to technology issues and the promotion of reform in mathematics, science and technology education.

Ronald A. Gove earned his Ph.D. in mathematics from Brandeis University, Waltham, MA, in 1972. He was employed as a cryptologic mathematician at the National Security Agency for 15 years and worked as a security consultant at Booz, Allen \& Hamilton until 1994. Until mid 1998 he was a Vice President and 
Operations Manager of the Center for Information Security Technology at Science Applications International Corporation. He is currently a Corporate Vice President at SAIC and is on the Group staff of the Software and Systems Integration Group.

\section{APPENDIX}

The basic structure of the model borrows from the SIR model in epidemiology, which classifies people as susceptibles (S), infectives (I) and removals or recovereds (R). This model and its extensions are commonly applied to so-called childhood immunizable diseases like measles in which susceptible people acquire the infection from contact with infective individuals who then recover to become immune from reinfection.

In adapting the model to analyze a single computer virus outbreak in an organization, the concepts of susceptibility, infection and immunity must be refined as shown in Figure 1. Contact means a behavior that allows the transmission of a computer virus to another computer, such as the distribution of a diskette or sending document (e.g., Microsoft Word) that contains a macro virus in the document's metadata. The intermediate copies of viruses, such as those on removable media like diskettes, are not explicitly counted. There may be some computers that have no defenses against the computer virus and are of course considered susceptible. However, some computers have defenses that are effective some of the time but not all of the time. For example, if antiviral software is effective but requires manual operation to scan potentially infected files, the degree of protection depends on the diligence of the user. These computers are classified as susceptibles that are partially immune and would be expected to have less exposure than a fully susceptible computer. As a special case, these computers may be totally immune and never allow infection. A computer virus infection terminates when it is detected, where the detected class is analogous to the recovered class in the SIR model. If the computer is fully susceptible, detection occurs when the user recognizes symptoms of the virus infection. If the computer is partially immune, detection is likely to occur through operation of antiviral software, although recognition of the symptoms of infection may also play a role. It is expected that the duration of infection among the partially immune computers will be considerably shortened because of the use of antiviral software. Detection of any infected computer is assumed to generate action that will halt the outbreak.

The model is based on a discrete time simulation having a daily time step. The actual construction of the model is done with the simulation package STELLA Research from High Performance Systems, Inc. STELLA Research is identical to HPS's iThink. The number of infections generated per time step depends on factors shown in Table 1 (see also Figure 1). The daily contact rate is the average number of contacts per day that could allow transmission of a computer virus from one computer to another in an outbreak. The fraction infective is the fraction of the 
computers that are already infective, aggregating computers with no immunity and with partial immunity. The checking frequency accounts for the fact that susceptible computers with partial immunity have reduced exposure depending on the likelihood that the software is actually used to check potential sources of infection when it is supposed to be used. The product, $(X I)(C)(F)$, is the average number of infections generated per time step among susceptibles with no immunity. The product, $(X 2)(C)(F)(I-K)$, is the average number of new infections generated per time step among susceptibles with partial immunity. The STELLA software package is used to construct a stochastic formulation by assigning the formula as the mean of a Poisson distribution.

Table 1 States and parameters of the model

$\begin{array}{ll}\text { Symbol } & \text { Description } \\ \mathrm{X} 1 & \text { Number of susceptibles with no immunity } \\ \mathrm{X} 2 & \text { Number of susceptibles with partial immunity } \\ \mathrm{Y} 1 & \text { Number of infectives with no immunity } \\ \mathrm{Y} 2 & \text { Number of infectives with partial immunity } \\ \mathrm{D} 1 & \text { Duration of infection with no immunity } \\ \mathrm{D} 2 & \text { Duration of infection with partial immunity } \\ \mathrm{M} & \text { Fraction partially immune } \\ \mathrm{C} & \text { Daily contact rate } \\ \mathrm{F} & \text { Fraction infective } \\ \mathrm{K} & \text { Checking frequency } \\ \mathrm{P} & \text { Compliance frequency }\end{array}$

The number of infections detected per time step also depends on factors shown in Table 1. The primary focus is on when the user detects problems in his or her own computer. The duration of infection corresponds to the time interval starting with the infection of a computer and terminating with the detection of the infection on that computer by the user. The average duration of infection would be expected to be rather short with partial immunity afforded by antiviral software and rather long when the user must recognize symptoms. If the duration of infection is a single day, then all infected computers are transferred to the detected class in one time step. Using the single day as a minimum duration, infections of longer duration result in a fraction of the infective computers being detected each day, corresponding to the inverse of the duration of infection after the first day is subtracted off. For infections with duration longer than a day, the STELLA software package is used to construct a stochastic formulation by assigning the ratio, $(Y 1) /(D l-1)$, for computers without immunity and the ratio, $(Y 2) /(D 2-1)$, for computers with partial immunity, as the mean of a Poisson distribution for the number of computers detected per time step. A more accurate distribution would be the Binomial, but a 
built-in variate with this distribution is not available in STELLA, so we have approximated the distribution with the Poisson. The Poisson distribution is a reasonable approximation to the Binomial when the daily probability of detection of an individual infected computer is low ( $20 \%$ or less) and the mean duration of infection is reasonably long (at least 5 days). In many computing environments, it is expected that the duration of infection is either a single day or several days long.

An additional process of notification may occur if users of uninfected computers detect the presence of a computer virus in someone else's computer because transmission of the computer virus was detected. This scenario assumes that susceptibles with partial immunity may detect transmission and then report the problem, thereby activating the same response as if the user of the infected computer had reported the problem. The number of infections notified in this way per time step depends on the compliance frequency, which represents the chance that a user who detects such an infection reports it properly. The product, $(X 2)(C)(F)(K)(P)$, is the average number of new infections notified per time step by susceptibles with partial immunity. The STELLA software package is used to construct a stochastic formulation by assigning the formula as the mean of a Poisson distribution.

Scenarios are defined by the selection of particular values of the parameters as shown in Table 2. In comparison to the baseline scenario, the coverage scenario increases the percentage of computers with antiviral software from $80 \%$ to $100 \%$, the efficacy scenario increases the checking frequency from $80 \%$ to $100 \%$ and the notification scenario increases the compliance frequency from $0 \%$ to $50 \%$. These three scenarios correspond to infection control strategies of improving immunization coverage, improving the efficacy of a vaccine, and improving early notification of infection, respectively. The last scenario for an undetectable computer virus corresponds to the situation when installed antiviral software is ineffective even when properly used.

The outbreak begins in a population of 100 computers with a single infective computer that may have no immunity or partial immunity. Each combination was iterated 100 times with a daily contact rate of 4 , a duration of infection of a single day if a computer has partial immunity and a mean duration of infection of 10 days if a computer has no immunity. The mean, minimum and maximum of the outbreak sizes (including the initial infective computer) are shown in Table 2 corresponding to the two different starting conditions. The minimum and maximum are shown in brackets following the mean outbreak size. The most obvious effect, of course, is that an absence of any effective antiviral software guarantees extensive spread of a computer virus once introduced. However, it is less obvious that transmission is reduced more by improving the distribution (coverage) of antiviral software than by improving the efficacy of antiviral software. Even more remarkable is the effect of a notification policy on reducing the spread of a computer virus without any improvements in the technology. 
Table 2 Mean, minimum and maximum outbreak sizes for different scenarios

\begin{tabular}{llllll} 
Scenario & $\begin{array}{l}\text { Fraction } \\
\text { partially } \\
\text { immune }\end{array}$ & $\begin{array}{l}\text { Checking } \\
\text { frequency }\end{array}$ & $\begin{array}{l}\text { Compliance } \\
\text { frequency }\end{array}$ & $\begin{array}{l}\text { Start with } \\
\text { no } \\
\text { immunity }\end{array}$ & $\begin{array}{l}\text { Start with } \\
\text { partial } \\
\text { immunity }\end{array}$ \\
\hline Baseline & 0.80 & 0.80 & 0 & $9.17[2,34]$ & $2.48[1,6]$ \\
Coverage & 1.00 & 0.80 & 0 & N/A & $1.84[1,5]$ \\
Efficacy & 0.80 & 1.00 & 0 & $7.86[1,20]$ & N/A \\
Notification & 0.80 & 0.80 & 0.50 & $3.59[1.15]$ & $2.46[1,7]$ \\
Undetectable & 0 & 0 & 0 & 53.16 & N/A \\
& & & & &
\end{tabular}

In order to better understand the effects of these strategies, a useful theoretical construct is the basic reproduction ratio, which represents the potential spread of infection from a single infected individual. The basic reproduction ratio characterizes threshold behavior of the dynamics of transmission. If the ratio is less than unity, then the infection does not spread. If the ratio is greater than unity, the infection does spread. Larger values of the ratio indicate a greater amount of spread.

Central to the construct of the basic reproduction ratio is the classification of random contacts at the initiation of an outbreak according to the immune state of the computer of the computer contacted as shown in Table 3. The approximate frequencies of each type of contact for the baseline scenario and the derivations are also shown in Table 3 (see also Table 1). The exact frequencies are slightly different because the first computer infected is removed from the susceptible population; the actual adjustment depends on the type of the first computer to be infected. As an outbreak progresses, the exact frequencies continue to change as the composition of the susceptible population changes.

Table 3 Frequencies of type of computer contact for baseline scenario

\begin{tabular}{lcc} 
Type of computer contact & Frequency & Derivation \\
\hdashline No antiviral software & $20 \%$ & $1-\mathrm{M}$ \\
Antiviral software not used effectively & $16 \%$ & $(\mathrm{M})(1-\mathrm{K})$ \\
Antiviral software used effectively & $64 \%$ & $(\mathrm{M})(\mathrm{K})$
\end{tabular}

The basic reproduction ratio is the product of contacts per day, the duration of infection, and the fraction of contacts that are susceptible as shown in Table 4. Note that, unlike the outbreak sizes shown in Table 2, the basic reproduction ratio does not include the initial infective computer. For all scenarios, the contact rate is the parameter described earlier. The other factors in the basic reproduction ratio require additional explanation. For each scenario, the fraction of contacts that is 
susceptible is derived from summing of the frequencies of the two types of contact shown in Table 3 that lead to infection. For the baseline scenario this is $20 \%+$ $16 \%$. The duration of infection is a weighted average of the mean duration of infection in a computer with no immunity (assumed to have a mean of 10 days) and the duration of infection in a computer with partial immunity (assumed to be a single day), where the weights are the relative frequencies of an infection in each category. For the baseline, among contacts that lead to infection, 56\% (20/36) is the chance that a computer has no immunity and $44 \%(16 / 36)$ is the chance that a computer has partial immunity. So the duration of infection is shown in Table 4 as $(56 \%)(10)+(44 \%)(1)$. The ratio of 8.7 means that an average of 8.7 infected computers would be expected from an initial infected computer. The coverage scenario drastically reduces the basic reproduction ratio to 0.8 by reducing the fraction of contacts that are susceptible and the typical duration of infection. In contrast, the efficacy scenario has only a modest effect on the basic reproduction ratio because computers without any antiviral protection continue to transmit for several days if infected. The effect of the notification policy comes entirely from changes in the duration of infection because infection is detected earlier if the initial infection is in a computer without immunity (the duration of infection in a computer with partial immunity cannot be reduced to less than a day). The reduction of the mean duration of infection from 10 days to 1.4 days for a computer without immunity is calculated from a Poisson process whose mean of 1.28 is the product of three factors: 4 contacts per day; $64 \%$ (the chance that a contact detects the infection); and $50 \%$ (the chance that someone who detects the infection notifies system administrators). The probability of no one notifying in a day is $\exp (-1.28)$, i.e. $\mathbf{2 8 \%}$, so that the daily probability of notification if there is an infected computer without immunity is $72 \%$ and the mean time till detection is $1 / .72=1.4$ days. For the notification scenario, the overall mean duration of 1.2 days is the weighted average allowing for infected computers with no immunity or partial immunity, where the weights are exactly the same as used in the baseline scenario.

Table 4 Basic reproduction ratios for different scenarios

\begin{tabular}{lllll}
$\begin{array}{l}\text { Scenario } \\
\text { contact }\end{array}$ & $\begin{array}{l}\text { Daily } \\
\text { (days) } \\
\text { rate }\end{array}$ & & $\begin{array}{l}\text { Fraction of } \\
\text { contacts } \\
\text { susceptible }\end{array}$ & $\begin{array}{l}\text { Basic } \\
\text { reproduction } \\
\text { ratio }\end{array}$ \\
\hdashline Baseline & 4 & $6.04(10 \times .56+1 \times .44)$ & 0.36 & 8.7 \\
Coverage & 4 & 1.0 & 0.20 & 0.8 \\
Efficacy & 4 & 10.0 & 0.20 & 8.0 \\
Notification & 4 & $1.2(1.4 \times .56+1 \times .44)$ & 0.36 & 1.7 \\
Undetectable & 4 & 10.0 & 1.00 & 40.0 \\
\hline
\end{tabular}




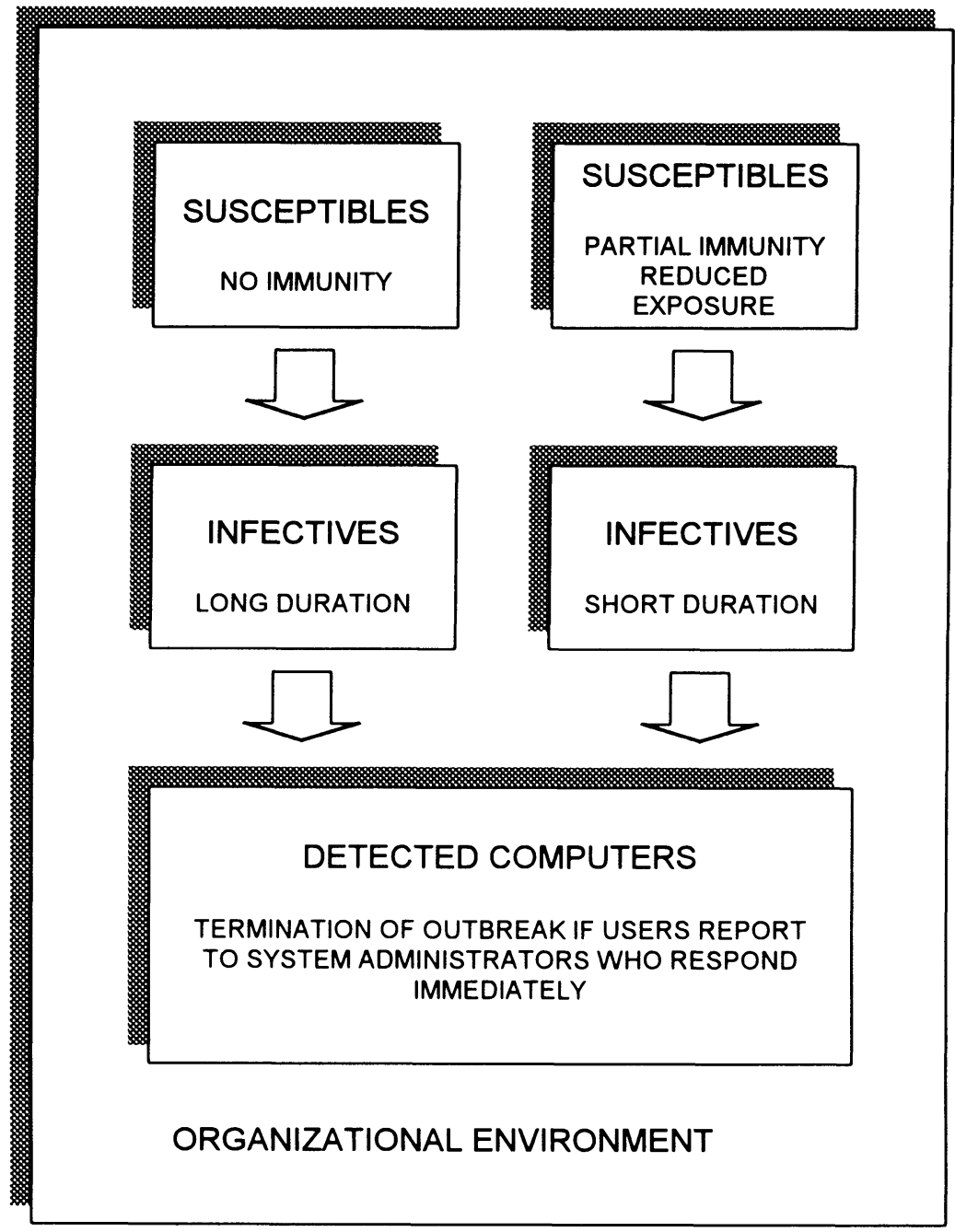

Figure 1 Epidemiological states of computer virus infection. 